\title{
SEMINARIO INTERNACIONAL LA TRADICIÓN CONSTITUCIONAL DE MÉXICO Y LA CONSTITUCIÓN DE 1917, DEL 17 AL 19 DE JUNIO DE 2015
}

\author{
Catherine Andrews \\ Centro de Investigación y Docencia Económicas (México)
}

El pasado junio se realizó el último seminario del proyecto La tradición constitucional de México y la Constitución de 1917, que la División de Historia del Centro de Investigación y Docencia Económicas organiza en el marco de su programa La Constitución de 1917 en su primer centenario.

El objetivo del proyecto es editar un libro de ensayos de investigación que estudian la relación de la Constitución de 1917 con la historia constitucional mexicana. Los trabajos de los investigadores participantes analizan la historia de los antecedentes, el contexto que propició la promulgación del texto constitucional de 1917, y su relación con las constituciones de los Estados que, en ese momento, componian la federación. En ellos, se busca contextualizar los debates constitucionales revolucionarios, de tal manera que florezcan tanto las continuidades como las rupturas del constitucionalismo mexicano con relación a su historia reciente, sobre todo en cuanto a las instituciones y prácticas instauradas por la Constitución de 1857 y el régimen porfirista.

El seminario se inauguró con la conferencia magistral de la Dra. María Refugio González (CIDE), titulado La Constitución de 1917. Las revoluciones y las reformas en los sistemas jurídicos. Se cerró con la conferencia magistral del Dr. Joaquín Varela Suanzes-Carpegna (Universidad de Oviedo) con el titulo: Algunas reflexiones metodológicas sobre la Historia Constitucional.

En la primera mesa, el tema de discusión fue la trayectoria de las primeras constituciones mexicanas de 1821 a 1856. Se presentaron dos ponencias:

Catherine Andrews (CIDE), Reformar la Constitución de 1824: Planes, proyectos y constituciones, 1824-1847.

Rosie Doyle (University of Warwick), Propuestas de Constitución, democracia y voluntad popular 1821- 1856.

Durante la segunda mesa, se habló de la historia de la Constitución de 1847, las leyes de Reforma y los proyectos conservadores de gobierno. Se presentaron tres ponencias:

Frédéric Johansson(Université de Paris 1), La Constitución de 1857: un texto renegado convertido en el simbolo del liberalismo.

Frédéric Johansson (Université de Paris 1), Las leyes de Reforma. El complemento revolucionario a la Constitución de 1857.

Georgina López González (UAM-Iztapalapa), Los proyectos constitucionales conservadores, 1857-1865. 
El tema de la tercera mesa fue la relación entre el constitucionalismo mexicano y el pensamiento católico. Se contó con las ponencias siguientes:

Pablo Mijangos (CIDE), Las relaciones Iglesia-Estado durante la vigencia de la Constitución de 1857.

Cecilia Bautista García (Universidad Michoacana de San Nicolás Hidalgo), Los congresos agrícolas católicos y los problemas sociales del campo mexicano: una contribución a la Constitución de 1917.

Yves Solís Nicot (Universidad Iberoamericana), El pensamiento católico frente a las Constituciones de 1857 y 1917.

Pablo Serrano Álvarez (E1 Colegio de Hidalgo), ¿Catolicismo social en la Constitución de 1917?

La cuarta mesa tuvo como tema los antecedentes inmediatos al Congreso Constituyente de Querétaro de 1917. Se presentaron cuatro ponencias. 1857.

Carlos Bravo Regidor (CIDE), Emilio Rabasa y las críticas a la Constitución de

Luis Barrón Córdova (CIDE), La Constitución de Coahuila como antecedente para el proyecto carrancista de 1917.

Miles Rodríguez (Bard College), La revolución zapatista, la reforma agraria y el artículo 27 de la Constitución de 1917.

En la quinta mesa se discutieron las instituciones de la Constitución de 1917. Hubo dos ponencias:

Ignacio Marván (CIDE), La reforma judicial en el constituyente de 1916-1917.

María Luna (UAM-Rectoría), Freno a la tirania: el presidencialismo en la Constitución de 1917.

La última mesa del seminario se desarrolló en torno al tema del impacto de la Constitución de 1917 en la vida política mexicana (1917-1940). Se presentaron las siguientes ponencias.

William Suárez-Potts (Kenyon College), El artículo 123: la incorporación de la legislación social en la Constitución de 1917.

Pavel Navarro (Museo de las Intervenciones) Obstáculos y avances para la propiedad de la Nación.

Ana María Serna Rodríguez (Instituto Mora), Libertad de expresión y delitos de imprenta en el marco legislativo revolucionario.

Timothy James (University of South Carolina) La administración de justicia y la Constitución de 1917.

Javier MacGregor Campuzano (UAM-Iztapalapa), Constitución, nueva ley electoral y viejas costumbres: calificación electoral y representación politica en los años veinte.

Se espera que los ensayos sean publicados en un libro colectivo durante el transcurso de 2016. 